\section{Geographische und ökonomische Aspekte der Inhalationstherapie}

\section{Einleitung}

Die Inhalation von Aerosolen zu therapeutischen Zwecken ist seit der Antike bekannt und besitzt heute in der Behandlung von Atemwegserkrankungen einen zentralen Stellenwert. Der grundsätzliche Vorteil einer topischen Therapie liegt in der hohen Konzentration am erkrankten Organ bei - absolut gesehen - geringen Medikamentenmengen, die nach der Resorption in niedrigen Konzentrationen im Gesamtorganismus durch die Verdünnung anfallen [12].

Jährlich werden weltweit ca. 500 Millionen Inhalationssysteme hergestellt. 74\% entfallen auf Dosieraerosole. Der Ersatz der fluorierten Kohlenwasserstoffe (FCKW) als Treibmittel stellt für die Industrie eine große Herausforderung dar. Erste Produkte mit alternativen Treibgasen (HFA 134a) wurden mittlerweile auf dem deutschen Markt eingeführt. Zunehmende Marktanteile gewinnen Pulverinhalationssysteme. An dritter Stelle sind preßluftgetriebene Düsenvernebler bzw. Ultraschallvernebler zu nennen. Die weltweiten Marktanteile im Jahre 1998 im Bereich der Asthmamedikation sind den Abb. 1 u. 2 zu entnehmen.

\section{Geographische Unterschiede im Verordnungsverhalten}

Bemerkenswerterweise gibt es erhebliche geographische Unterschiede in der Verordnung von Medikamenten z. B. in der Therapie des Asthma bronchiale [26]. In Großbritannien und Australien beträgt der Anteil inhalativer Steroide und BetaMimetika $80 \%$, in den USA hält sich die orale und die inhalative Medikation die Waage. Japan stand 1980 in der Gesamtverordnung von Beta-Mimetika (inhalative und orale Medikation) an 3. Stelle hinter den USA und Großbritannien, der Anteil der Inhalationssysteme lag dort nur bei 30\% [23]. In Asien sind kulturelle Faktoren zu berücksichtigen. Nur von der oralen Applikation erwartet man sich eine ausreichende Wirkung, daher stellt Theophyllin in 50\% die erste Wahl in der Therapie dar. Dennoch stieg die Verordnung z.B. von Salbutamol von ca. 100000 Kanistern im Jahr 1980 auf 2,5 Mio 1995 an, den gleichen Aufwärtstrend verzeichneten die inhalativen Steroide.

In Australien und Großbritannien liegen die Ausgaben für alle Asthmamedikamente (ausgedrückt in Behandlungsmonaten

Pneumologie 53 (1999) 612-616

(c) Georg Thieme Verlag Stuttgart · New York

\section{P. Haidl, D. Köhler}

Krankenhaus Kloster Grafschaft, Zentrum für Pneumologie, Beatmungs- und Schlafmedizin, Schmallenberg-Grafschaft

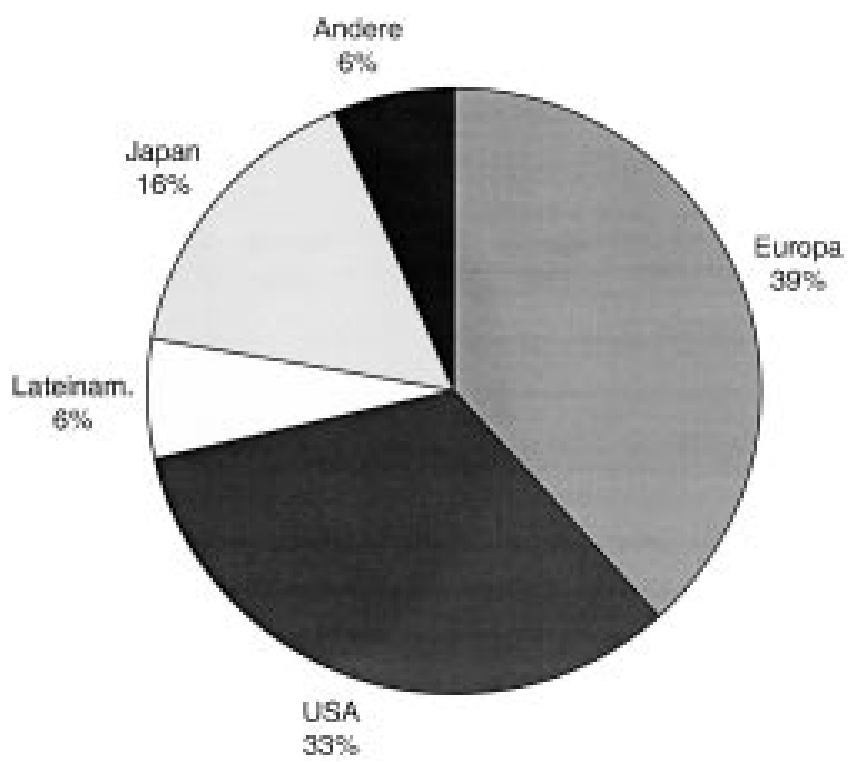

Abb. 1 Anteile der umsatzstärksten Regionen am Weltmarkt der Asthmamedikamente (oral und inhalativ) nach den IMS-Statistiken der pharmazeutischen Industrie.

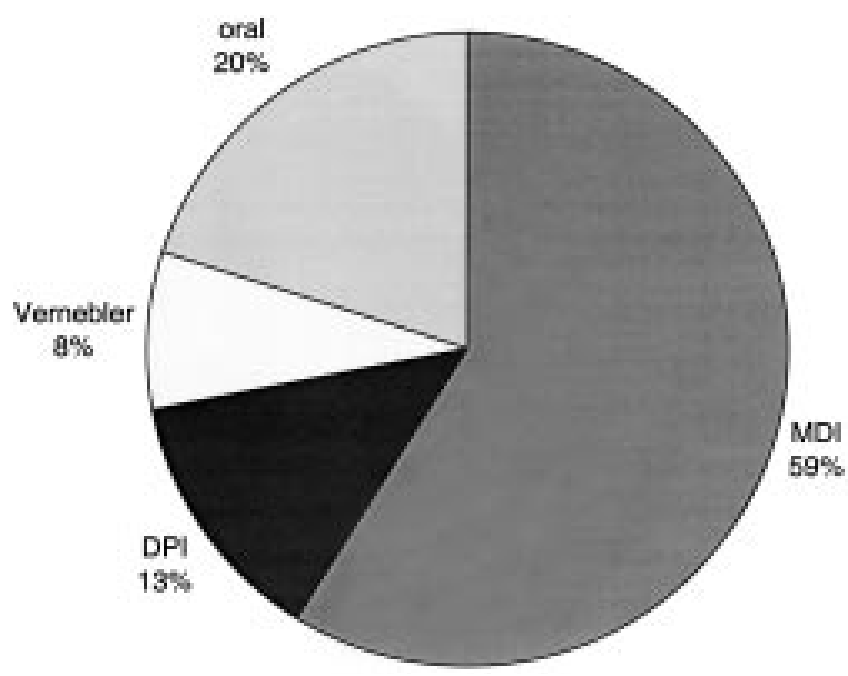

Abb. 2 Anteile der unterschiedlichen inhalativen Applikationssysteme und der oralen Gabe am Weltumsatz von 10,1 Mill. US \$ (Quelle wie 2). MDI metered dose inhaler, DPI dry powder inhaler. 
pro 1000 Einwohner) um den Faktor 3 höher als z.B. in den USA, Japan oder Frankreich [26]. Über die Ursachen kann nur spekuliert werden. Neben der höheren Asthmaprävalenz kann dies Ausdruck einer fortschrittlichen Therapie mit dem frühzeitigen Einsatz inhalativer Steroide sein. Eine Befragung von Pneumologen („Welches Medikament setzen Sie als erstes in der Therapie des Asthma bronchiale ein?") zeigte, daß 92\% der Briten, aber nur 32\% der Franzosen mit einem inhalativen Steroid begannen [26]. Ebenso große Unterschiede ergaben sich im Gebrauch vom Peak-Flow-Meßgeräten und der Weitergabe von Notfallplänen an die Patienten. Bei der Interpretation der Zahlen sind nicht allein medizinische Faktoren zu berücksichtigen. In den USA wurden bisher von den Krankenversicherungen die Kosten für Verneblersysteme und Inhalationslösungen erstattet, aber nicht für Atemwegs-Medikamente in Form eines Dosieraerosols oder Pulversystems. In Japan senkt die Verwendung des preiswerten Theophyllins die Kosten. Frankreich setzt auf eine starke Preiskontrolle des Marktes, der dadurch zumindest teilweise weniger attraktiv für die pharmazeutische Industrie ist.

Geographische Differenzen sind auch in der Verordnungshäufigkeit von Dosieraerosolen im Vergleich zu Pulversystemen zu erkennen. Skandinavien und die Niederlande weisen einen hohen Marktanteil der Trockenpulver im Gegensatz zu den englischsprachigen Ländern und Deutschland auf. Dabei ist zu berücksichtigen, daß in den Niederlanden bis vor kurzem Dosieraerosole im Gegensatz zu Pulversystemen von den Krankenkassen nicht erstattet wurden. In Skandinavien ist ein Marktführer in der Produktion von Pulversystemen ansässig, was den hohen Marktanteil erklärt.

Zahlen zur regionalen Verteilung der Verwendung von Verneblern liegen nicht vor. Dies hängt mit unterschiedlichen Vergütungssystemen zusammen. In Deutschland und den USA werden Vernebler von den Krankenkassen direkt erstattet. In anderen Ländern (z. B. Norwegen und teilweise Schweden) werden Verneblersysteme nur über zentrale Hilfsmittelstellen an Patienten ausgeliehen, was die Verfügbarkeit für die Patienten einschränkt. In Frankreich erhalten Ultraschallvernebler einen Marktvorteil, da die Vermietung dieser Geräte den Apothekern viel besser vergütet wird als das Ausleihen eines Düsenverneblers.

Eine umfangreiche europäische Studie [10] untersuchte die Unterschiede im Gebrauch antiasthmatischer Medikamente (inhalative und orale) in 14 Ländern. In allen Ländern wiesen Raucher eine deutlich niedrigere Wahrscheinlichkeit der Anwendung auf. Alter und sozioökonomischer Status beeinflußten die Häufigkeit der Medikation nicht. Nur in Frankreich, Irland und Italien nahmen Frauen mit signifikant höherer Wahrscheinlichkeit Medikamente.

Zusammenfassend wird die Auswahl des Inhalationssystems in den verschiedenen Ländern neben den medizinischen Aspekten stark durch kulturelle und historische Faktoren (z. B. Tradition eines Kurwesens mit Inhalationstherapie in der BRD) sowie das Erstattungsverhalten der Versicherungsträger beeinflußt.

\section{Compliance}

Ein zentraler Punkt in der Bewertung einer Methode zur Applikation von Medikamenten ist die Akzeptanz durch den Patienten, die dann die Mitarbeit (Compliance) im Therapiekonzept beeinflußt. Fehlende Compliance gilt als bedeutsamer Faktor in der Morbidität und Mortalität des Asthmas [7]. Andererseits ist eine $100 \%$-Compliance fern jeder Realität. Bei chronischen Erkrankungen, z.B. der arteriellen Hypertonie [18], geht man von ca. 50\% der Patienten aus, die ihre Medikation in effektiver Dosis einnehmen.

In bezug auf das klinische Ergebnis gibt es unterschiedliche Formen der ungenügenden Therapietreue: z.B. der Patient mit Asthma, der sein Therapieregime während einer Exazerbation einhält, danach aber jede Medikation absetzt oder derjenige, der stets nur einen kleinen Teil des Therapiekonzeptes umsetzt (,little bit each day“). Zu berücksichtigen sind Ängste vor Medikamentenabhängigkeit, Wirkungsverlust bei Dauergabe und die Abneigung gegenüber synthetisierten, „unnatürlichen“ Substanzen [15].

Wird die Therapietreue durch das inhalierte Medikament beeinflußt? Nach Inhalation eines Beta-Mimetikums erhält der Patient mit einem Asthma bronchiale durch die verspürte Bronchodilatation eine Rückmeldung über die korrekte Inhalation. Auch bei schlechter Koordination zwischen Sprühstoßauslösung und Inhalation kann durch wiederholte Anwendung eine oft ausreichende Wirkung erzielt werden. Nachteil hierfür sind aber höhere Therapiekosten. Anders verhält es sich bei der Inhalation von Steroiden. Die richtige Applikation kann nur indirekt über den längerfristigen klinischen Verlauf (u.a. Peak-Flow-Monitoring, Anzahl symptomfreier Tage) beurteilt werden. Zumindest nimmt der Patient nach Auslösung des Sprühstoßes einen Nebel wahr. Bei Pulverinhalatoren ohne Zusatz eines Hilfsstoffes (in der Regel Lactose) besteht das Manko, daß gar nicht erkannt wird, ob Wirkstoff freigesetzt wurde. Bei fehlerhafter Inhalation von Steroiden, insbesondere mittels Dosieraerosol ohne Verwendung eines Hohlraumsystems, kommt es nicht nur zum Wirkungsverlust, sondern es steigt auch die Nebenwirkungsrate. Grund ist die vermehrte oropharyngeale Deposition mit nachfolgender systemischer Resorption sowie die Zunahme von Soor und Stimmbanddysfunktion im Sinne von Heiserkeit.

In der Grassic-Studie [16] (Befragung von 320 Pat. in NordostSchottland) ergab sich nun aber kein Unterschied in der Einstellung der Patienten gegenüber Beta-Mimetika oder Steroiden. Der stärkste Prädiktor der Abneigung gegen inhalative Steroide war die Ablehnung von Beta-Mimetika. Bei dieser Befragung kam auch zum Ausdruck, daß insbesondere Männer darunter leiden, bei einer Inhalation in der Öffentlichkeit gesehen zu werden. Ebenso unangenehm werden pfeifende Atemgeräusche empfunden, d. h. Symptome, die die Erkrankung der Umgebung signalisieren.

In einer englischen Studie \{3] wurde untersucht, ob die Compliance der inhalativen Steroidtherapie durch eine fixe Kombination mit einem Beta-Mimetikum in einem Pulverinhalationssystem gesteigert werden kann. Bei den 72 Patienten ergab sich aber kein Vorteil gegenüber der getrennten Applikation der beiden Substanzen. Nur die Hälfte der Patienten inhalierte mehr als 80\% der verordneten Dosen. Dabei ist zu 
berücksichtigen, daß allein die Teilnahmebereitschaft an einer Studie therapietreuere Patienten selektioniert. Vor kurzem wurde eine fixe Kombination von Salmeterol und Fluticason auf dem deutschen Markt eingeführt. Es wird interessant sein, den Einfluß auf die Compliance zu verfolgen.

In einer aufwendigen amerikanischen Untersuchung von 59 Pat. [9] erfüllten nur 25\% der Inhalationsmanöver alle geforderten 4 Kriterien einer korrekten Anwendung eines Dosieraerosols. Auffallend war die Geschlechtsdifferenz. Zwar inhalierten $43 \%$ der Männer korrekt, aber nur 4\% der Frauen. Nun ist die Asthmaprävalenz bei Frauen und Männern gleich. Es gibt Daten, daß aber die Anzahl der Notaufnahmen im Krankenhaus bei Frauen doppelt so hoch ist. Ein Zusammenhang zwischen fehlerhafter Inhalationstechnik und Instabilität der Erkrankung bietet sich an. Unterstellen könnte man auch einen Einfluß des Alters und der Erkrankung („der junge Patient mit Asthma inhaliert zuverlässiger als der ältere Patient mit chronisch obstruktiver Bronchitis“). Hier ergaben sich jedoch in dieser Untersuchung [9] keine signifikanten Unterschiede.

Gibt es Hinweise, daß psychologische Charakteristika die Therapietreue beeinflussen? Bosley [2] untersuchte $102 \mathrm{~Pa}$ tienten in bezug auf ihre Compliance bei der Inhalation von Terbutalin und Budesonid. Anschließend wurden psychologische Fragebogen ausgefüllt und semistrukturierte Interviews geführt. Die Patienten mit einer niedrigen Compliance hatten einen signifikant höheren Depressions-Score. Eine Erklärung boten die Autoren nicht an. Möglicherweise mindert die innere Leere bzw. die Antriebslosigkeit des depressiven Patienten die Bereitschaft zu einer regelmäßigen Medikamentenanwendung. Auch der Gedanke „mir kann ja doch nicht geholfen werden“ mag eine Rolle spielen. In der Kategorie Angst ergab sich kein Unterschied zwischen den Gruppen. Die Autoren diskutierten, daß beim Asthma bronchiale der Grad der Angst generell sehr hoch sei. Angst kann positive (Motivation zur Therapie) als auch negative (lähmende Angst) Auswirkungen in bezug auf die Compliance haben.

Eine weitere Überlegung ist, ob die Compliance abhängig vom Schweregrad des Asthmas sein kann. Mann u. Mitarb. [14] gingen dieser Frage nach und fanden keinen Zusammenhang. Es mehren sich aber die Hinweise, daß mit Zunahme des Schweregrades sogar eher die Bereitschaft abnimmt, einer Exazerbation mit einer Therapieintensivierung zu begegnen. Andererseits soll ein höherer Schweregrad an Luftnot die
Compliance erhöhen [25]. Zur Klärung dieser Frage sind weitere Untersuchungen notwendig.

Knapp tausend Patienten mit chronisch obstruktiver Lungenerkrankung, die zu Hause eine Vernebler-Therapie durchführten, wurden in eine therapieadhärente, d.h. mit hoher Compliance (50,6\%) und eine nicht adhärente Gruppe (49,4\%) eingeteilt [25]. Signifikante Korrelationen zur hohen Compliance ergaben sich u.a. für die Dauer der Schulausbildung, für verheiratete Patienten, Nichtraucher und Patienten mit einem Theophyllinspiegel über $9 \mu \mathrm{g} / \mathrm{ml}$ (Tab. 1). Diese Ergebnisse decken sich nicht ganz mit der eingangs zitierten europäischen Studie [10]. Hier beeinflußte der sozioökonomische Status in verschiedenen Länder die Compliance nicht, identisch war aber der Bezug zwischen Rauchgewohnheiten und geringer Therapietreue. Bosley u. Mitarb. [1] untersuchten ebenfalls die Compliance bei Anwendung einer Verneblertherapie. Zur Kontrolle wurde ein Drucksensor im Verneblerkopf verwendet, so daß das alleinige Einschalten des Gerätes nicht genügte. Die mittlere Compliance betrug 56,8\%. 36 der 93 Patienten (44\%) wurden als compliant eingestuft, d. h. sie hielten mehr als 70\% der verordneten Inhalationen ein.

Compliance hängt weiter eng mit der Anzahl der verabreichten Medikamente zusammen. Cramer u. Mitarb. [8] fanden, daß die Compliance eines Therapiekonzeptes mit zweimaliger Gabe $81 \%$ betrug und auf $39 \%$ bei viermaliger Applikation sank. So sollten Therapievorschläge stets unter diesem Gesichtspunkt durchforstet werden. Kelloway [11] überprüfte Unterschiede zwischen einer oralen Theophyllintherapie und einer inhalativen antientzündlichen Therapie (Steroid oder DNCG). Gerade bei Jugendlichen ergab sich eine mittlere Compliance der oralen Therapie von $70 \%$ gegenüber $30 \%$ bei Steroidinhalation. Daraus ergibt sich der Stellenwert einer oralen Therapie. Ideal wäre eine Asthmatherapie mit einer einzigen Tablette als Basis. Ein Hoffnungsträger in dieser Hinsicht sind die Leukotrienantagonisten. Ihr Einsatz wird bei mildem bis mittelschwerem Asthma überprüft $[5,13,21]$. Ein aktueller Beitrag zur Vereinfachung von Therapieplänen wird die bereits erwähnte Einführung einer fixen Kombination eines Steroids mit einem langwirksamen Beta-Mimetikum in einem Pulversystem sein.

Abschließend weist die Problematik „Compliance“ in der Inhalationstherapie mehr unbeantwortete Fragen als Antworten auf. In der Einschätzung der Compliance eines Patienten sind der Raucherstatus und das Vorhandensein einer depres-

\begin{tabular}{|c|c|c|c|}
\hline \multirow[b]{2}{*}{ Anzahl der Schuljahre* } & \multirow{2}{*}{$\begin{array}{l}\text { niedrige Compliance } \\
\mathrm{n}=487 \\
10,4 \pm 3,5\end{array}$} & \multicolumn{2}{|c|}{$\begin{array}{l}\text { hohe Compliance } \mathrm{p} \\
\mathrm{n}=498\end{array}$} \\
\hline & & $10,9 \pm 3,2$ & 0,033 \\
\hline Anzahl Verheirateter & $291(60)$ & $355(71)$ & 0,001 \\
\hline Raucher & $234(48)$ & $159(32)$ & 0,001 \\
\hline Dyspnoe-Score* & $2,7 \pm 0,7$ & $2,9 \pm 0,7$ & 0,001 \\
\hline $\begin{array}{l}\text { Patienten mit Theophyllin-Spiegel } \\
>9 \mu \mathrm{g} / \mathrm{ml}\end{array}$ & $144(31)$ & $217(45)$ & 0,001 \\
\hline $\begin{array}{l}\text { Benutzungsdauer (min) } \\
\text { des Verneblers/Tag* }\end{array}$ & $12,5 \pm 7,5$ & $40,6 \pm 13,0$ & 0,001 \\
\hline
\end{tabular}

Tab. 1 Vergleich der Patienten mit hoher und niedriger Compliance ihrer häuslichen Verneblertherapie (modifiziert nach [22])

\footnotetext{
* Mittelwerte \pm SD, Zahlenwerte in Klammern sind Prozentangabe.
} 
siven Grundstruktur von Bedeutung. Die Zahl der Dosen pro Tag sollte so gering wie möglich gehalten werden.

\section{Kosten-Nutzen-Relation}

Der Wunsch nach einer möglichst wirksamen Therapie zu einem wirtschaftlich vertretbaren Preis bestimmt zunehmend unser medizinisches Denken. Zu berücksichtigen ist, daß 70\% der Patienten mit Asthma bronchiale in die Gruppe „mildes Asthma“ gehören und 20\% der Gesamtkosten verursachen [4]. Die $10 \%$ der Patienten mit schwerem Asthma tragen zu über $50 \%$ an den Gesamtaufwendungen bei. Davon entfallen ca. $44 \%$ auf die Kosten, die durch die stationäre Behandlung entstehen.

Damit sind Interventionen (z.B. Einführung neuer Medikamente) in der letzteren Gruppe bezüglich der Kosten-NutzenRelation effizienter. Bei Patienten mit einem milden Asthma kann eine neue Substanz nur dann kostenneutral oder -sparend sein, wenn sie einen unmittelbaren dramatischen Effekt hat oder wenigstens einen geringeren positiven Einfluß über einen langen Zeitraum aufweist. Ökonomisch und medizinisch wertvoll sind insbesondere Therapiestrategien, die ein Fortschreiten eines leichtgradigen Asthma bronchiale in ein schwereres und damit teureres Stadium verhindern.

Die Kosten können in direkte (Ausgaben für Prävention und Therapie) und indirekte (durch die Krankheit bedingte Fehlzeiten am Arbeitsplatz, vorzeitige Sterblichkeit und Erwerbsunfähigkeit) unterteilt werden [16,22]. Der Anteil variiert stark von Land zu Land, auch abhängig von unterschiedlichen Berechnungsmethoden. In Australien beträgt der Anteil der direkten Kosten 77\%, in den USA 54\% und in Schweden nur $43 \%$. Schweden weist einen hohen Prozentsatz an Kosten für eine ambulante Therapie auf, in den USA verbraucht die stationäre Behandlung den Großteil der Geldmittel. Pro Patient mit Asthma bronchiale und Jahr betragen die volkswirtschaftlichen Kosten in den USA 640 \$, am unteren Rand liegt Australien mit $326 \$$, Spitzenreiter ist Schweden mit 1315 \$ [22]. Das Beispiel Australien zeigt, daß trotz hoher Ausgaben für die medikamentöse Therapie [26] die Gesamt-

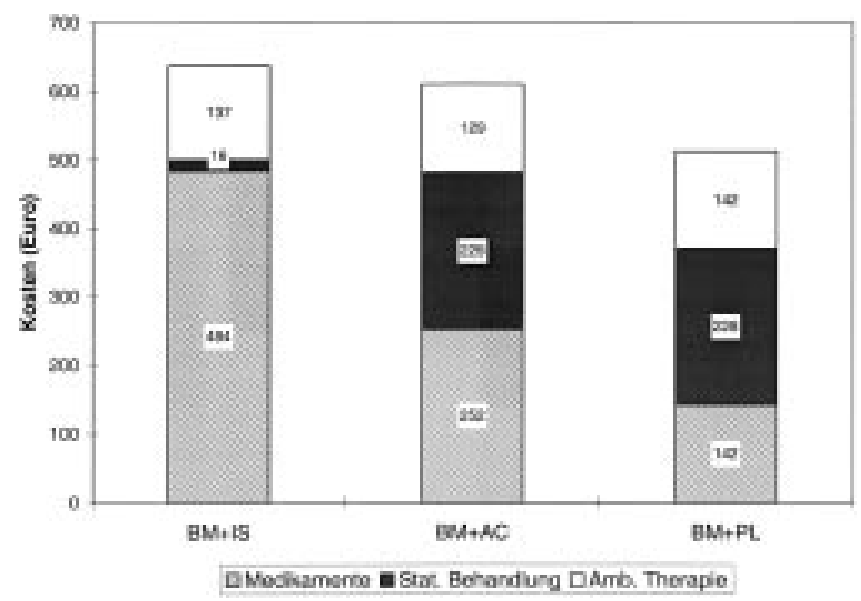

Abb. 3 Verwendete Medikamente: Beta-Mimetikum (BM), inhalatives Steroid (IS), Anticholinergikum (AC), Plazebo (PL) (modifiziert nach [19]). kosten durch erheblich geringere indireke Kosten fallen können. In einer sorgfältigen Untersuchung konnte Nowak Daten für Deutschland erheben [15]. Die Gesamtausgaben pro Jahr für die Erkrankung Asthma bronchiale lagen im Jahr 1992 bei 5130 Mio. DM, die direkten Kosten betrugen 61,4\%. Die Kosten pro Patient und Jahr beliefen sich auf umgerechnet ca. $750 \$$ und überschritten knapp das US-Niveau. Daten aus der Schweiz ergaben das identische Verhältnis direkter zu indirekter Kosten, die Gesamtkosten pro Patient und Jahr lagen mit ca. $1700 \$$ um den Faktor 2,3 höher. Die Autoren boten keine Erklärung dieser enormen Differenz an [20]. Bei den direkten Kosten führten die Ausgaben für eine stationäre Behandlung mit $46 \%$ vor den Aufwendungen für Medikamente ( $41 \%$ ), die ambulanten Arztkosten betrugen $6 \%$.

In einer über 2,5 Jahre angelegten niederländischen Studie [19] wurden 274 Patienten mit moderater chronisch obstruktiver Lungenerkrankung (alle wiesen zusätzlich eine bronchiale Hyperreagibilität auf) untersucht. Verglichen wurden die klinische Wirksamkeit sowie die Kosten dreier verschiedener Therapiearme: a) Beta-Mimetikum (BM) und inhalatives Steroid, b) BM und ein Anticholinergicum, c) BM und Plazebo. Die Therapie mit dem inhalativen Steroid erhöhte signifikant die Anzahl der symptomfreien Tage und senkte den Grad der Hyperreagibilität. Die Gesamtkosten nahmen um 28\% im Vergleich zur Plazebogruppe zu. Allerdings sanken innerhalb des Gesamttopfes die Ausgaben für die ambulante Therapie sowie für eine stationäre Behandlung deutlich (Abb. 3). Die Mehrkosten pro Patientenjahr betrugen 201 \$ (180 Euro) im Vergleich zur Plazebogruppe. Dies muß gegen die höhere Stabilität der Erkrankung abgewogen werden. Gesamtwirtschaftlich wird die Rechnung günstiger ausfallen, da hierbei die Kostenreduktion durch weniger Arbeitsausfälle einfließt.

In diesem Zusammenhang muß die große Bedeutung der Patientenschulung genannt werden. Durch eine strukturierte und evaluierte Asthmaschulung werden Kosten gespart [24]. Signifikant sinken die Fehltage am Arbeitsplatz, die stationären Behandlungstage und die Zahl der schweren Asthmaanfälle. Zwar bleibt der Medikamentenverbrauch gleich, aber die gleichen Medikamente werden nun unter Berücksichtigung des Therapiestufenschemas effizienter eingenommen. $1 \mathrm{DM}$, die für die Schulung aufgewendet wird, spart der Gesellschaft den Betrag von 5 DM [24].

Zusammenfassend gilt für die inhalative Therapie, daß die sorgfältige Instruktion des Patienten, aber auch die regelmäßige Überprüfung der Inhalationstechnik Grundvoraussetzung für eine medizinisch wirksame und ökonomische Therapie sind. Leider trägt die Vielzahl der Systeme mehr zur Verwirrung von Therapeuten und Patienten bei als zur Therapieoptimierung. Wichtig ist, die Patientenpräferenzen in die Auswahl des Inhalationssystem einzubeziehen [6]. Dem Patienten sollte sowohl ein Dosieraerosol als auch ein Pulversystem demonstriert werden (im Einzelfall ebenso ein Vernebler) und bei der Entscheidung müssen die individuellen Fähigkeiten einfließen. Aufgrund zahlreicher patientenspezifischer Besonderheiten kann einem Inhalationsprinzip alleine nicht das Prädikat: „System mit der höchsten Compliance“ verliehen werden. Für die Compliance ist nicht entscheidend, ob das Inhalationsssystem dem Arzt zusagt, sondern dem Patienten. 


\section{Fazit}

Geographische Unterschiede in der Inhalationstherapie weisen auf Motive und Präferenzen im Verordnungsverhalten hin. Diese stehen im Zusammenhang mit den gesamtwirtschaftlichen Kosten, die bei der Behandlung (z. B. Inhalationstherapie) einer Erkrankung (z.B. Asthma bronchiale) entstehen. In bezug auf die Effizienz der Aufwendungen und die Reduktion der Ausgaben besitzt die Verbesserung der Patientencompliance einen hohen Stellenwert.

\section{Literatur}

${ }^{1}$ Bosley CM, Corden ZM, Rees PJ, Cochrane GM. Psychological factors associated with use of home nebulized therapy for COPD. Eur Respir J 1996; 9: 2346 - 2350

2 Bosley CM, Fosbury JA, Cochrane GM. The psychological factors associated with poor compliance with treatment in asthma? Eur Respir J 1995; 8: 899 - 904

${ }^{3}$ Bosley CM, Parry DT, Cochrane GM. Patient compliance with inhaled medication: Does combining beta-agoniste with corticosteroids improve compliance?. Eur Respir J 1994; 7: 504 - 509

${ }^{4}$ Buist AS. Pros and cons of oral anti-asthma medication. Eur Respir Rev 1995; 5(28): 158 - 160

${ }^{5}$ Chanarin N, Johnston SL. Leukotriens as a target in asthma therapy. Drugs 1994; 47(1): $12-24$

${ }^{6}$ Chapman KR. Die Wahl des geeigneten Inhalationssystems im Erwachsenen- und Kindesalter. J Aerosol Med 1995; 8(Suppl 2): $25-33$

${ }^{7}$ Cochrane GM. Therapeutic compliance in asthma; its magnitude and implications. Eur Respir J 1992; 5: 122 - 125

${ }^{8}$ Cramer JA, Mattson RH, Prevey ML, Scheyer RD, Quellette VL. How often is medication taken as prescribed? J Am Med Assoc 1989; 261: $3272-3277$

${ }^{9}$ Goodman ED, Israel E, Rosenberg M, Johnston R, Weiss TS, Drazen MJ. The influence of age, diagnosis and gender on proper use of metered-dose inhalers. Am J Respir Crit Care Med 1994; 150: $1256-1261$

${ }^{10}$ Janson C, Chinn S, Jarvis D, Burney P. Individual use of antiasthmatic drugs in the European community respiratory health survey. Eur Respir J 1998; 12: 557 - 563

${ }^{11}$ Kelloway JS, Wyatt SA, Adlis SA. Comparison of patients' compliance with prescribed oral and inhaled asthma medications. Arch Intern Med 1994; 154: 1349 - 1352

12 Köhler D, Fleischer W. Was ist gesichert in der Inhalationstherapie. München: Arcis; 1988

${ }^{13}$ Leff JA, Busse WW, Pearlmen D et al. Montelukast, a leukotrienereceptor antagonist, for the treatment of mild asthma and exercise-induced bronchoconstriction. N Engl J Med 1998; 339: $147-152$

${ }^{14}$ Mann MC, Eliasson O, Patel K, Zuwallack RL. An evaluation of severity-modulated compliance with q.i.d. dosing of inhaled beclomethasone. Chest 1992; 102: 1342 - 1346

${ }^{15}$ Marinker M. The current status of compliance. Eur Respir Rev 1998; 8(56): 235 - 238

${ }^{16}$ Nowak D, Volmer T, Wettengel R. Asthma bronchiale - eine Krankheitskostenanalyse. Pneumologie 1996; 50: 364 - 371

17 Osman LM, Russell IT, Friend JAR, Legge JS, Douglas JG. Predicting patient attitudes to asthma medication. Thorax 1993; 48: 827 830

18 Rudd P. Clinicians and patients with hypertension: unsettled issues about compliance. Am Heart J 1995; 130: 572 - 579

${ }^{19}$ Rutten-Van Mölken M, Doorslaer van E, Jansen M et al. Costs and effects of inhaled corticosteroids and bronchodilators in asthma and chronic obstructive pulmonary disease. Am J Respir Crit Care Med 1995; 151: 975 - 982

${ }^{20}$ Szucs TD, Anderhub H, Rutishausen M. The economic burden of asthma: direct and indirect costs in Switzerland. Eur Respir J 1999; 13: 281 - 286

${ }^{21}$ Spector LS, Smith LJ, Glass M. Accolate ${ }^{\mathrm{TM}}$ Asthma Trialists Group: Effects of 6 weeks of therapy with oral doses of ICI 204,219, a leukotriene D4 receptor antagonist, in subjects with bronchial asthma. Am J Respir Crit Care Med 1994; 150: 618 - 623

22 Sullivan SD. Economics of asthma and asthma treatments. Eur Respir Rev 1998; 8(59): 351 - 355

${ }^{23}$ Takishima T. Current status and trends of aerosol inhalation diagnosis and therapy in Japan. J Aerosol Med 1999; 12: 1 - 8

24 Trautner C, Richter B, Berger M. Cost-effectiveness of a structured treatment and teaching programme on asthma. Eur Respir J 1993; 6: 1485 - 1491

${ }^{25}$ Turner J, Wright E, Mendella L, Anthonisen N. Predictors of patients adherence to long-term home nebulizer therapy for COPD. Chest 1995; 108: $394-400$

${ }^{26}$ Vermeire P. Differences in asthma management around the world. Eur Respir Rev 1994; 4(21): 279 - 281

\section{Dr. Peter Haidl}

Krankenhaus Kloster Grafschaft 57392 Schmallenberg-Grafschaft

E-mail: p.haidl@t-online.de 